\title{
Proteinosis alveolar pulmonar
}

\author{
PATRICIO RIOSECO S.*
}

Pulmonary alveolar proteinosis an update

Pulmonary Alveolar Proteinosis (PAP) is a rare disease characterized by the accumulation of surfactant derived lipoproteinaceous material filling the alveoli, secondary to failure of its clearance by macrophages. Most of the patients are adults that have auto antibodies directed to Granulocyte-Macrophage Colony Stimulating Factor (GM-CSF). The evolution is towards disturbed gaseous exchange with a wide spectrum of disease from spontaneous recovery to death. There are three forms of PAP: genetic, secondary and autoimmune. Symptoms are scarce and patients may present with dyspnea, dry cough and hypoxemia. Chest $X$ ray shows bilateral opacities and thorax CT depicts ground glass opacities surrounded by septal widening, the so called "crazy paving” pattern. Diagnosis is made on clinical and radiological grounds and confirmed by PAS positive staining of bronchoalveolar lavage material or surgical lung biopsy. Accepted treatment is whole lung lavage (WLL) with saline. Alternatives are subcutaneous or inhaled GM-CSF, Plasmapheresis or Rituximab, and even modification of the method of WLL and combination of different manner of treatment.

Key words: Pulmonary Alveolar Proteinosis; Granulocyte macrophage colony stimulating factor; Pulmonary Surfactants; Bronchoalveolar Lavage; Plasmapheresis; Rituximab.

\section{Resumen}

La Proteinosis Alveolar Pulmonar (PAP) es una enfermedad poco frecuente, caracterizada por la acumulación de material lipoproteico derivado del surfactante pulmonar al interior de los alvéolos por una falla de depuración de este material por los macrófagos alveolares, siendo la causa más frecuente de esta disfunción la acción bloqueadora producida por anticuerpos anti factor estimulante de colonias de granulocitos y macrófagos (GM-CSF) lo que lleva a un deterioro del intercambio gaseoso. La evolución es variable abarcando desde la resolución espontánea hasta la insuficiencia respiratoria grave y la muerte. Se describen tres formas de PAP: Genética, secundaria y autoinmune (antes primaria o idiopática) siendo esta última la más frecuente en adultos. Clínicamente, se manifiesta por disnea, tos seca e hipoxemia que pueden ser progresivas. En la radiografía de tórax se encuentran opacidades bilaterales y la tomografía computarizada de tórax de alta resolución (TACAR) muestra vidrio esmerilado con sobre posición de engrosamiento septal intra e interlobulillar, patrón conocido como "crazy paving”. El diagnóstico se basa en la clínica y en el lavado broncoalveolar con material PAS positivo. La biopsia quirúrgica es confirmatoria. El tratamiento clásico es el lavado pulmonar total (LPT) para remover el contenido alveolar. Otras alternativas son la administración de GM-CSF subcutáneo o inhalado, plasmaferesis y rituximab, cuyos resultados son variables. Diferentes autores han modificado la forma del LPT y combinado los diferentes métodos de tratamiento con el fin de obtener resultados más rápidos y efectivos.

Palabras clave: Proteinosis Alveolar Pulmonar; Factor Estimulante de Colonias de Granulocitos y Macrófagos; Surfactante pulmonar; Lavado broncoalveolar; Plasmaféresis; Rituximab.

* Unidad de Enfermedades Respiratorias Hospital Las Higueras. Talcahuano, Chile. 


\section{Introducción}

La PAP es una enfermedad extremadamente infrecuente. Las tasas de prevalencia e incidencia comunicadas en la literatura son de 3,7 hasta 40 por millón de habitantes y 0,2 por millón de habitantes respectivamente esto, basado principalmente en comunicaciones de casos y de algunas series $^{1}$. Revisando la literatura global, Huaringa y Francis encuentran hasta el año 2016 tan solo 363 casos publicados ${ }^{2}$.

En nuestro país no existen estadísticas al respecto y solo hemos encontrado dos publicaciones de casos; uno en un niño y el otro en un adulto ${ }^{3,4}$. En el presente número de esta revista, Rioseco y colaboradores comunican un caso adulto con características especiales en cuanto a su evolución y tratamiento ${ }^{5}$. Dada la tasa de incidencia mencionada, es muy probable que existan unos 8.500 casos en el país y que un neumólogo chileno solo vea uno o dos casos durante su vida activa.

La enfermedad llamó la atención por primera vez en 1953 cuando Castelman comentó de un paciente en el cual los alvéolos pulmonares estaban llenos de material proteináceo. Se le consideró una nueva enfermedad. Fue descrita y comunicada en 1958 por Rosen, quien describió los hallazgos en 27 pacientes cuya característica común era el relleno alveolar con material proteináceo. De allí el nombre de proteinosis alveolar pulmonar. La gran mayoría de estos enfermos falleció por insuficiencia respiratoria y muchos estaban cursando infecciones oportunistas o no habituales. El tratamiento era empírico y consistía en administrar mucolíticos, antibióticos y corticoesteroides en altas dosis y por períodos prolongados con los consiguientes efectos secundarios a los cuales se responsabilizó por las infecciones ${ }^{6,7}$. No hubo innovaciones en la terapia hasta que en 1963, Ramírez Rivera describió por primera vez la técnica y racionalidad del uso del lavado pulmonar como tratamiento de la PAP, utilizando el método de canular cada pulmón en forma sucesiva instilando alícuotas de solución salina varias veces al día ${ }^{8}$. Dos años después, el mismo investigador modificó el procedimiento utilizando por primera vez un tubo endotraqueal de doble lumen a través del cual instilaba solución salina mezclada con acetil cisteína y heparina en cada pulmón en forma secuencial. La técnica fue exitosa y mejoró la sintomatología y la radiología de los pacientes en que se utilizó ${ }^{\text {. Con algunas }}$ modificaciones, dependiendo del centro en que se realice, hasta el momento, el tratamiento de elección de la enfermedad sigue siendo el lavado pulmonar total (Figura 1).

\section{Etiopatogenia}

El origen último de la enfermedad no se conoce, pero en su etiopatogenia está la acumulación de proteínas y lípidos del surfactante pulmonar al interior de los alvéolos secundaria a una falla en la depuración de este material por los macrófagos alveolares. La causa más frecuente de esta disfunción es la acción bloqueadora producida por autoanticuerpos anti Factor Estimulante de Colonias de Granulocitos Macrófagos (GM-CSF).

El surfactante pulmonar es producido por los neumocitos tipo II y se compone principalmente de fosfolípidos en un 80 a $90 \%$ siendo los más importantes la fosfatidil colina (corresponde al $70 \%$ de los lípidos, en forma de dipalmitoil fosfatidil colina), el fosfatidil glicerol y el fosfatidil inositol más cuatro proteínas del surfactante: A, B, C y D. Las proteínas A y D son hidrofílicas y son miembros de la familia de proteínas inmunes innatas o colectinas ${ }^{10}$. Se ha encontrado sitios de unión para las colectinas en la superficie de virus y bacterias por lo que ellas participan en la inmunidad innata y adaptativa ${ }^{11,12}$. Por otra parte, también a estas proteínas se les ha descrito una función microbicida ${ }^{13,14}$. Las proteínas B y C, altamente hidrofóbicas, se encuentran asociadas a los lípidos formando una estructura de propiedades biofísicas muy importantes. Tal estructura permite que los lípidos secretados por los neumocitos tipo II, en especial la dipalmitoil fosfatidil colina (DPPC) se dirijan a la interfase aire/líquido de la superficie alveolar. El extremo hidrofílico de la DPPC está determinado por el nitrógeno cuaternario de la colina que se orienta hacia la fase líquida de la interfase aire-líquido, en tanto que las colas hidrofóbicas de la DPPC constituidas por sus dos moléculas de ácido palmítico apuntan hacia la fase aérea de la interfase y hace que la estructura fosfolipídica-proteica se disponga en una monocapa cuya función es disminuir la tensión superficial alveolar en función del tamaño de los alvéolos. Si consideramos a los alvéolos como esferas con su superficie interna cubierta por una delgada capa de líquido, según la ley de Laplace su presión estaría determinada por la tensión superficial (TS) y su radio (r) de acuerdo a la siguiente fórmula: Presión $=2 \mathrm{TS} / \mathrm{r}$. Si la tensión superficial de la interfase aire-líquido alveolar se mantuviera constante durante el ciclo respiratorio, en la espiración los alvéolos más pequeños tendrían una mayor presión y se colapsarían vaciándose en los de mayor tamaño, este colapso pulmonar no ocurre ya que el surfactante disminuye la tensión superficial alveolar en espiración homogenizando la relación $\mathrm{TS} / \mathrm{r}$, con lo 
cual la presión interna de los alvéolos es similar en espiración y el trabajo respiratorio es menor.

De lo anterior se deduce que la principal función del material surfactante pulmonar es la de reducir la tensión superficial a nivel de la interface aire/líquido de los alveolos impidiendo su colapso al final de la espiración; sus funciones secundarias son modular las respuestas inmunes a nivel pulmonar y prevenir la diseminación de gérmenes al interactuar con ellos y eventualmente eliminarlos ${ }^{10}$.

En condiciones normales, los neumocitos tipo II sintetizan el surfactante en el retículo endoplásmico, es modificado a nivel del aparato de Golgi y almacenado como cuerpos lamelares para ser secretados por exocitosis hacia la superficie alveolar en forma de una malla de mielina tubular que como antes se dijo, a nivel de la interface aire/líquido se dispone en monocapa formando un film que disminuye la tensión superficial.

Una vez inactivado este material por la compresión de la pared alveolar durante la espiración, los complejos lipoproteicos son desplazados de la superficie de este film, siendo degradados por los macrófagos o reciclados por los neumocitos tipo II para ser reutilizados ${ }^{15}$. Se estima que entre el 25 al $95 \%$ del surfactante es reciclado siendo re-internalizado en los cuerpos lamelares y resecretados ${ }^{16}$. Si el equilibrio entre producción y degradación del material surfactante pulmonar se rompe, necesariamente habrá alteraciones tanto por déficit como es en el caso del Síndrome de Distrés Respiratorio del Recién Nacido, como por exceso a nivel de las estructuras profundas del pulmón como sucede en la PAP, lo que determinará alteraciones en el intercambio gaseoso y eventualmente insuficiencia respiratoria. En este equilibrio juegan un papel fundamental los ma- crófagos alveolares, los que a su vez como células residentes del territorio, cumplen además una labor de defensa de primer orden. El GM-CSF, es una citoquina que estimula la diferenciación, proliferación y sobrevida de células mieloides, monocitos, neutrófilos y células dendríticas de modo que la disminución o el bloqueo del receptor de esta citoquina en el macrófago, llevará a disfunción de esta estirpe celular, tanto en su función de depuración del material surfactante como en su función microbicida. Por esta razón, los pacientes que sufren de PAP, son susceptibles a infecciones por gérmenes no habituales ${ }^{5,6,14}$.

Existen tres formas por las cuales se puede acumular material del surfactante en los alvéolos: una forma congénita que se asocia a mutaciones recesivas en los genes SFTPB Y SFTPC que codifican las proteínas $\mathrm{B}$ y $\mathrm{C}$ o en las subunidades alfa o beta del receptor de activación del GM-CSF transformándolo en disfuncional. Cada una de estas subunidades corresponde a los genes csf2ra y csf2rb. Las mutaciones en el primero son más frecuentes y se ven en niños, desde el recién nacido hasta los 9 años. Las mutaciones en csf $2 \mathrm{rb}$, poco frecuentes se han comunicado en individuos de hasta 36 años de edad. Esta forma de PAP es la menos frecuente, representa alrededor del $1 \%$ de los casos y no se encuentran autoanticuerpos ni en el líquido de lavado alveolar ni en el suero ${ }^{17-19}$. Hay una forma secundaria asociada a cualquier condición o enfermedad que disminuya la población funcional de macrófagos en el territorio alveolar. Este grupo, que corresponde a más o menos el $4 \%$ de los casos se asocia a enfermedades hematológicas especialmente a los síndromes mielodisplásticos, tratamientos inmunosupresores e infecciones (VIH, Mycobacterium tuberculosis) siendo de pésimo

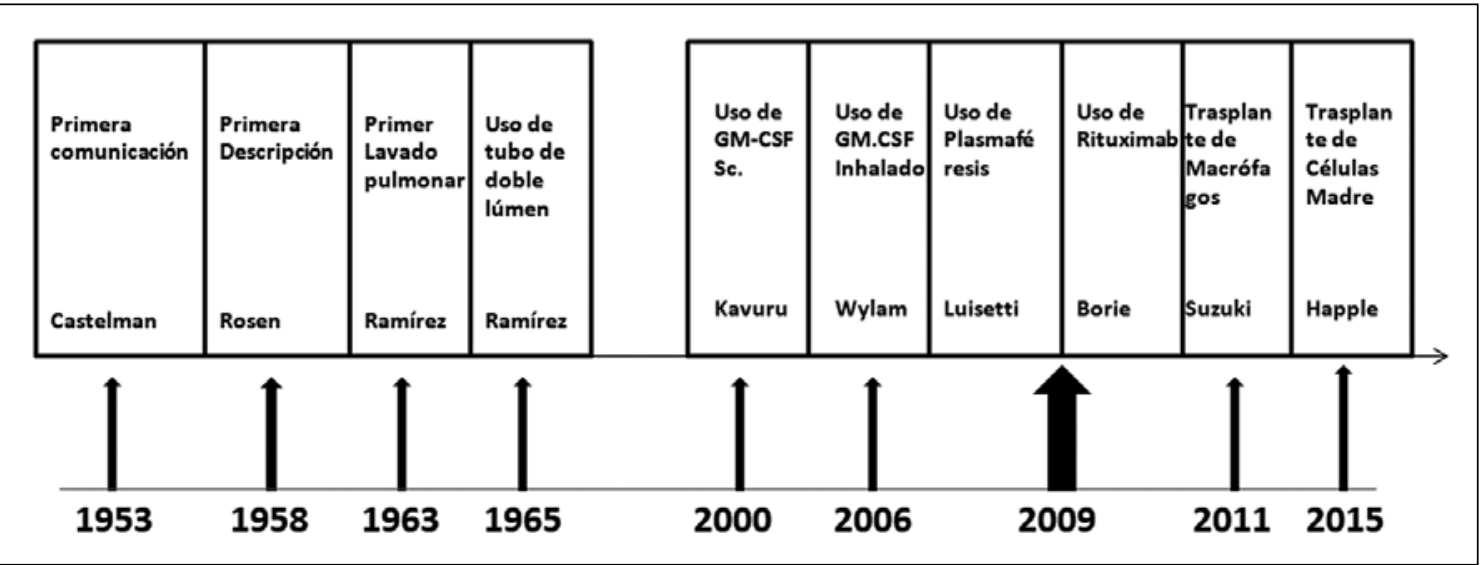

Figura 1. Principales hitos en el conocimiento de la Proteinosis alveolar. Se muestra la evolución de las modificaciones en el tratamiento de la PAP desde su primera comunicación en 1953 hasta nuestros días. 
pronóstico. Tampoco aquí se encuentran autoanticuerpos. Por otra parte, es probable que la inhalación de polvos industriales, especialmente sílice, titanio, aluminio, induzca la aparición de autoanticuerpos como es el caso por ejemplo de la sílice que se asocia a los autoanticuerpos de la artritis reumatoidea, lupus eritematoso sistémico, esclerodermia y glomerulonefritis ${ }^{19}$ y podría considerarse que en el caso de la PAP, la inhalación laboral de polvos, indujera la aparición de anticuerpos anti GM-CSF, pero tal asociación no es clara, ya que existen estudios japoneses de pacientes expuestos a polvos industriales (no precisados en la publicación) que desarrollan PAP, pero sin la presencia de auto anticuerpos ${ }^{19-23}$. Entre la inhalación crónica de los muchos polvos descritos como asociados a PAP están los fertilizantes como un raro factor. En cuanto a la exposición aguda a tóxicos, se describe un cuadro con características clínicas similares a la PAP en individuos expuestos a la inhalación masiva de sílice $^{22}$. Finalmente está la forma idiopática o autoinmune que corresponde a cerca del $98 \%$ de los casos en la cual se encuentran anticuerpos anti GM-CSF (del tipo IgG) tanto en la circulación general como a nivel alveolar ${ }^{23}$. Las tres formas de PAP se asocian a disfunción de los macrófagos alveolares lo que lleva a una alteración de la homeostasis del surfactante alveolar con la consiguiente acumulación de material lipoproteináceo al interior de los alvéolos, que se traduce finalmente en alteraciones del intercambio gaseoso con falla respiratoria hipoxémica que puede progresar hasta causar la muerte. La enfermedad no es entonces una enfermedad intersticial con las cuales se suele confundir, sino más bien una enfermedad de relleno alveolar.

\section{Clínica}

Nos referiremos principalmente a la PAP autoinmune por ser la más frecuente de las presentaciones.

La relación hombre/mujer es de 2:1. El 56\% de los pacientes son fumadores o exfumadores siendo el hábito más frecuente en el sexo masculino; la edad promedio al momento del diagnóstico es de 51 años, pero se ha comunicado casos de hasta 72 años. Alrededor del 33\% de los pacientes son asintomáticos al momento del diagnóstico y este constituye un hallazgo durante el estudio de otras patologías o exámenes laborales.

La sintomatología es inespecífica siendo el motivo de consulta más frecuente la disnea de evolución lenta y progresiva en el 39\% de los casos; en un $21 \%$ de los casos se ha reportado tos habitualmente seca. El examen físico puede ser completamente normal aunque entre un $30 \%$ y un $50 \%$ de los pacientes se encuentra crepitaciones finas en las bases pulmonares, cianosis e hipocratismo digital ${ }^{20}$.

La presencia de fiebre, dolor torácico y hemoptisis casi nunca están presentes, pero es necesario considerar que en alrededor de un 5\% de los pacientes con PAP confirmada, se encuentra infecciones oportunistas siendo las más frecuentes, Nocardia (43\%) y M. tuberculosis (37\%), seguidas por histoplasmosis, aspergilosis, criptococosis $(20 \%)$ y otras en las cuales la etiología no puede ser precisada ${ }^{24}$.

\section{Diagnóstico}

En razón a lo paucisintomático del cuadro, es necesaria una evaluación desde el punto de vista laboral y probable exposición a tóxicos; radiológica; serológica; hematológica; funcional; endoscópica y eventualmente histopatológica. Se trata de clasificar al paciente en uno de los tres tipos de PAP ya que el pronóstico y el enfrentamiento terapéutico pueden ser diferentes.

Desde el punto de vista laboral, es importante la exposición a polvo de sílice (mineros, areneros, limpieza de barcos en diques secos), titanio, aluminio, polvos de madera y cereales (empresas forestales), talco, cemento y caolín. En una serie francesa, el 39\% de adultos con PAP tuvieron algún tipo de exposición inhalatoria a tóxicos ${ }^{25}$. La radiografía de tórax muestra habitualmente opacidades perihiliares simétricas, mayormente hacia las bases en disposición de "alas de murciélago", similar a lo que se observa en el edema pulmonar, pero sin cardiomegalia ni derrame pleural. No hay disminución del volumen pulmonar como en las enfermedades intersticiales. Es muy frecuente que la sintomatología del paciente no se compadezca con lo impresionante de la imagen radiológica.

La tomografía computarizada d alta resolución (TACAR) de tórax. es el examen que sugiere con más fuerza el diagnóstico de PAP. Se observa un patrón de vidrio esmerilado secundario al relleno alveolar con material lipoproteináceo y engrosamiento septal, adoptando el característico patrón de "crazy-paving" o empedrado que predomina hacia las zonas inferiores. Sin embargo, esta imagen no es en absoluto patognomónica de PAP, pues también se suele ver en edema pulmonar cardiogénico, SDRA (síndrome de distrés respiratorio agudo), hemorragia alveolar e infecciones (Pneumocistis jirovesii) aunque en la PAP no hay 
nódulos, quistes, adenopatías mediastínicas ni zonas de consolidación ${ }^{26,27}$.

La bioquímica sanguínea habitual en general es normal. En el 50\% de los casos, la LDH se encuentra elevada así como también el antígeno carcino-embrionario y los niveles de la glicoproteína KL-6. Estos marcadores junto a alteraciones funcionales respiratorias, en la capacidad de difusión para el monóxido de carbono (DLCO) y en la diferencia alvéolo-arterial de $\mathrm{PO}_{2}$, aunque no sirven para el diagnóstico, son útiles como puntaje de gravedad de la enfermedad ${ }^{22}$. Los niveles séricos de las proteínas A, B y D del surfactante se encuentran elevados y se les puede considerar también como índice de severidad. Con el tratamiento, la única proteína que varía en su concentración es la $\mathrm{D}$ por lo que se le podría utilizar en el seguimiento de un paciente tratado ${ }^{20}$. La detección de anticuerpos del tipo IgG anti GMCSF por ELISA es el gold standard y se puede realizar tanto en líquido de lavado alveolar como en el suero. La detección de anticuerpos por medio de técnicas de aglutinación en látex tiene un $100 \%$ de sensibilidad y un $98 \%$ de especificidad y niveles superiores a $2,8 \mu \mathrm{g} / \mathrm{ml}$ son consistentes con el diagnóstico ${ }^{1}$.

En el estudio funcional respiratorio son importantes, la espirometría, que entre el $10 \%$ y $30 \%$ de los casos suele ser normal, pero la mayoría muestra un patrón restrictivo. En fumadores puede haber una alteración obstructiva con CVF disminuida. La DLCO que está disminuida en el $40 \%$ al $50 \%$ de los casos con un gradiente alvéolo-arterial de $\mathrm{PO}_{2}$ aumentado y una fracción de shunt medida respirando oxígeno al $100 \%$ aumentada $^{23}$. En 1/3 de los casos hay hipoxemia de reposo, pero en más del $50 \%$, se produce una caída de la $\mathrm{PaO}_{2}$ con el ejercicio. Una serie rumana documenta el hecho de que todos los pacientes en quienes se realizó el test de caminata de $6 \mathrm{~min}$ (TC6M) tuvieron una desaturación de la hemoglobina (evaluada por oximetría) de entre un $4 \%$ y un $28 \%$ desde la línea basal ${ }^{28}$.

La endoscopía es útil para realizar un lavado broncoalveolar. El líquido recuperado luego de la instilación de solución salina es característicamente de aspecto lechoso, turbio y sedimenta rápidamente. Mandatorio para el diagnóstico es la tinción del líquido con Ácido Peryódico de Schiff (PAS) y el examen citológico. Se observan macrófagos alveolares espumosos que contienen gránulos eosinófilos y material hialino extracelular que se tiñe en forma homogénea con PAS. El estudio ultraestructural demostrará la presencia de numerosos cuerpos lamelares ${ }^{24}$.

En general el diagnóstico se hace con la ima- gen tomográfica, el lavado broncoalveolar y la detección de anticuerpos séricos, pero en casos en que con ello no se llegue a diagnóstico, es necesario recurrir a la histología. La biopsia idealmente se debe hacer en forma quirúrgica ya que la biopsia transbronquial es diagnóstica solo en el $42 \%$ de los casos. Existe una comunicación de la utilidad de la criobiopsia broncoscópica en un enfermo con lavado broncoalveolar negativo ${ }^{1,4}$. Una nueva técnica comunicada por un grupo francés ha utilizado endomicroscopía confocal con fluorescencia que podría ser de utilidad en pacientes demasiado graves como para soportar una biopsia quirúrgica o endoscópica ${ }^{29}$.

En la macroscopía el pulmón es firme y nodular y al corte escurre un líquido de aspecto opaco lechoso. La histología muestra el relleno del espacio alveolar con un material acelular, granular, eosinófilo que habitualmente se tiñe positivamente con PAS y se detecta conservación de la arquitectura pulmonar.

\section{Tratamiento}

El tratamiento aceptado de la PAP es el lavado pulmonar total. Sin embargo, esta aproximación no se dirige a la causa de la enfermedad sino a su consecuencia, es decir, a eliminar la acumulación de exudado alveolar lipoproteináceo liberando así unidades alveolares que no estaban participando en el proceso de intercambio gaseoso para mejorar la falla respiratoria. El procedimiento no está estandarizado, así como tampoco está definido el momento en la evolución de la enfermedad en que se indica. Así por ejemplo, una encuesta global realizada en 27 centros de Europa, Asia, Sudáfrica y Estados Unidos, revela que entre los 17 centros que trataban solo adultos, el $82 \%$ realiza el lavado pulmonar total con el paciente intubado con un tubo de doble lumen, anestesiado con drogas endovenosas y ventilado mecánicamente, el resto, hace lavado segmentario o lobar broncoscópico con el paciente sedado. Entre los 7 centros que trataban solo niños, la mayoría hacía lavado segmentario o lobar broncoscópico y solo uno, realizaba lavado pulmonar total. Finalmente, en 3 centros que atienden una población mixta de adultos y niños, solo en uno se realizaban las dos formas de lavado pulmonar. El análisis demostró que la forma de lavado broncoscópico se reserva en general para aquellos pacientes que por su gravedad no pueden tolerar el lavado pulmonar total para niños muy pequeños en los cuales es imposible canular la vía aérea con un tubo de doble lumen ${ }^{31}$. La enfermedad puede remitir en forma espontánea en solo un 10\% de los casos ${ }^{31}$, 
de modo que, el lavado pulmonar total debe estar disponible cuando nos enfrentamos a un paciente con PAP. La decisión de utilizarlo debe basarse en la sintomatología especialmente si el paciente sufre de disnea en reposo, $\mathrm{PaO}_{2}$ menor de 65 $\mathrm{mmHg}$, diferencia alvéolo-arterial de $\mathrm{PO}_{2}$ mayor de $40 \mathrm{mmHg}$ o desaturación en el TC6M o si la función respiratoria se deteriora progresivamente. El procedimiento más aceptado es realizado bajo anestesia general endovenosa. A través de un tubo endotraqueal de doble lumen se aísla un pulmón y mientras el otro se ventila se instila por gravedad cantidades diversas, pero en promedio, de $800 \mathrm{ml}$ de solución salina calentada a $37^{\circ}$, y con el apoyo de kinesioterapia percutoria, se aspira o deja salir por gravedad. El procedimiento se repite tantas veces como sea necesario para que el líquido recuperado sea claro. En general esto dura alrededor de dos a seis horas. En promedio, se usan $14 \mathrm{~L}$ por cada pulmón. Importante es tener en cuenta la capacidad dialítica de la solución de lavado y la membrana alveolar de modo que el control frecuente de electrolitos especialmente potasio es mandatorio, De la misma manera, se debe monitorizar la temperatura corporal, ya que es frecuente la hipotermia y controlar la oxigenación por las alteraciones de la relación ventilación/perfusión que determina el llenado de líquido de un pulmón con la consiguiente compresión vascular y desplazamiento de la circulación hacia el pulmón seco y hacia zonas de pulmón mejor ventiladas. El lavado del pulmón contralateral se realiza en algunos centros en la misma ocasión o a las $48 \mathrm{~h}$, pero en el $50 \%$ de los casos este se lleva a cabo a la semana o a las dos semanas siguientes ${ }^{31}$. La mejoría de los síntomas ocurre en el $85 \%$ de los casos en forma casi inmediata y la mejoría radiológica y funcional, en los días o semanas siguientes. El paciente es extubado a las pocas horas o al día siguiente y puede egresar para planificar el lavado contralateral en otra oportunidad. Existe un $70 \%$ de pacientes que requerirán de un segundo lavado pulmonar dentro de los próximos 5 años siendo el promedio de la duración en la mejoría sintomática de 15 meses. Un porcentaje de los pacientes que vuelve a tener síntomas se puede seguir solo con controles clínicos, radiológicos y funcionales hasta que eventualmente requieran de un nuevo lavado. Un $10 \%$ de los pacientes no responden a esta terapia ${ }^{32}$.

Un enfoque que se dirige más hacia la etiopatogenia de la enfermedad es la administración de GM-CSF vía subcutánea, endovenosa o inhalatoria. Los primeros ensayos en que se usó GM-CSF datan del año 2000, en los cuales se administró
GM-CSF por vía subcutánea en dosis de $250 \mu \mathrm{g}$ pudiéndose aumentar a 9 y $18 \mu \mathrm{g} / \mathrm{kg} /$ día si había una buena tolerancia al fármaco. 12 de 25 pacientes mostraron una mejoría en la $\mathrm{PaO}_{2}$ en niveles similares a la obtenida por lavado pulmonar total. Sin embargo, esta mejoría fue más lenta. Los efectos adversos fueron mayormente locales, en el sitio de inyección ${ }^{33}$. Mejor efecto se ha logrado con el uso de GM-CSF por vía inhalatoria utilizando dosis de $250 \mu \mathrm{g}$ cada $12 \mathrm{~h}$. En un estudio retrospectivo 11 de 12 pacientes mejoraron clínica y funcionalmente ${ }^{35}$. Un estudio prospectivo de Tazawa y cols realizado en 35 pacientes mostró que el $68 \%$ de ellos mejoraban ya desde el período inicial de inducción de la droga de 6 semanas sin que se lograra demostrar cambios en los niveles séricos de anticuerpos anti GM-CSF. Los efectos secundarios fueron mínimos, pero se desconoce por cuánto tiempo debe mantenerse la terapia, aun cuando en el estudio de Wylam, dos pacientes en los cuales se obtuvo remisión completa de la enfermedad, se encontraban libres de esta a los 2 años de suspendido el tratamiento ${ }^{34,35}$. En 9 estudios tanto con la droga subcutánea como por vía inhalatoria, los tiempos de tratamiento varían entre 4 semanas y 48 semanas $^{36}$.

Tampoco existen marcadores biológicos o clínicos que predigan en qué paciente la terapia será efectiva y actualmente este tratamiento debe considerarse como una alternativa al lavado pulmonar total y no como terapia primaria.

Aún más etiológicas son las investigaciones dirigidas a eliminar los anticuerpos anti GM-CSF como el uso de Rituximab, plasmaféresis y el trasplante de precursores hematopoyéticos o de macrófagos.

El Rituximab es un anticuerpo monoclonal que se dirige al receptor antigénico CD20 de superficie de los linfocitos B. Su uso produce un descenso importante de los linfocitos B circulantes y por lo tanto una rápida depleción de las células presentadoras de antígenos, afectando la población de células plasmáticas productoras de anticuerpos. Es racional entonces su uso en una enfermedad que depende de la presencia de autoanticuerpos como la PAP. Hay comunicaciones de casos en que se obtuvo mejoría, en pacientes que rechazaron el lavado pulmonar total o que fallaron a este, con recuperación de la oxigenación y los hallazgos radiológicos ${ }^{37,38}$. En un estudio abierto fase II en el cual 10 pacientes con PAP recibieron dos infusiones de $1.000 \mathrm{mg}$ de rituximab cada 15 días se obtuvo una mejoría significativa de los niveles de $\mathrm{PaO}_{2}$, diferencia alvéolo-arterial de $\mathrm{PO}_{2}$ y puntaje de la tomografía computada de tórax con respecto a la basal a los tres y seis 
meses en todos los pacientes, lo que se correlacionó con la disminución de los anticuerpos anti GM-CSF en el líquido de lavado broncoalveolar, pero no con sus niveles séricos ${ }^{39}$. Aún cuando los resultados son prometedores, tampoco se puede considerar un tratamiento de primera línea y se le debe considerar solo cuando el paciente es refractario a la terapia habitual.

La plasmaféresis podría ser efectiva en retirar anticuerpos anti GM-CSF circulantes. Hay comunicaciones de su uso en casos aislados en los cuales el tratamiento habitual y el uso de factor estimulante de colonias de granulocitosmacrófagos (GM-CSF) había fracasado y se ha reportado la disminución del título de anticuerpos sérico con mejoría clínica ${ }^{40,41}$. En 2015 Garber y colaboradores describen el protocolo utilizado en un paciente refractario al lavado pulmonar total, uso de GM-CSF exógeno y rituximab a quien trataron con 5 sesiones de plasmaféresis en días consecutivos, seguidos de nueva administración de rituximab obteniendo una muy significativa disminución del título de anticuerpos anti GMCSF junto con la mejoría clínica y funcional del enfermo $^{42}$.

Existen comunicaciones de trasplante de macrófagos maduros o de los progenitores de los macrófagos solo en animales de experimentación con persistencia de los mismos, multiplicación y función adecuada al instilarlos por vía endotraqueal en modelos de PAP hereditaria en ratas las cuales mejoran los parámetros clínicos y biológicos de la enfermedad. Podría considerarse el inicio de estudios clínicos en el futuro cercano en este tipo de PAP ${ }^{43-45}$.

La Figura 1 muestra un resumen de los hechos principales de la evolución en el tiempo desde la primera descripción de la PAP a la actualidad.

El trasplante pulmonar es considerado como una opción en casos excepcionales en que todas las alternativas en uso han fracasado y la vida del paciente se encuentra severamente amenazada. Hay una serie de trasplante pulmonar en niños en la cual el $6,3 \%$ fue por $\mathrm{PAP}^{46}$ con la posible recurrencia de la enfermedad en el pulmón trasplantado.

En lo referente a la técnica del lavado pulmonar total, hay autores que han modificado la forma habitual de realizarlo con el objetivo de eliminar en mejor forma el contenido alveolar, así por ejemplo, Bonella examina la cinética de la eliminación del contenido proteico por espectrometría del líquido recobrado, comparando la técnica habitual con la aplicación de ventilación manual del pulmón lavado durante el procedimiento y demuestra una mejor depuración con la modificación introducida, lo cual podría acortar la duración del mismo ${ }^{47}$. En otros casos, utilizando los conocimientos adquiridos durante los ensayos de la ventilación parcial líquida con perfluorocarbonos, especialmente Perflubron, han utilizado este líquido, solo o en conjunto con surfactante sintético cuando el lavado tradicional con solución salina falla 5 .

Los perfluorocarbonos son un tipo de hidrocarburos en los cuales se ha reemplazado el hidrogeno por flúor y en el caso del Perflubron, se agrega un átomo de bromo. Su fórmula química es $\mathrm{C} 8 \mathrm{BrF} 17$. Es una substancia líquida incolora, bioquímicamente inerte ya que la unión $\mathrm{C}-\mathrm{F}$ es extremadamente firme, tiene mayor densidad que el agua $(1,3 \mathrm{~g} / \mathrm{ml})$ muy baja tensión superficial (18 dinas $/ \mathrm{cm})$, alto coeficiente de propagación, baja presión de vapor, 20 veces la solubilidad para el $\mathrm{O}_{2}(57 \mathrm{ml} / 100 \mathrm{ml})$ y el $\mathrm{CO}_{2}(210 \mathrm{ml} / 100$ $\mathrm{ml}$ ) al compararlo con la solución salina y 2 veces la de la sangre, es estable, no soluble en agua y con un alto peso específico. Se absorbe pobremente y se elimina principalmente por evaporación. Dada la alta densidad del compuesto, se distribuye fácilmente en las zonas dependientes del pulmón y en teoría, podría abrir unidades alveolares colapsadas; desplazar los detritus y productos inflamatarios y, posiblemente el exudado lipoproteináceo de la PAP los cuales flotan en la superficie de la fase líquida desplazada por el producto permitiendo su más fácil remoción por aspiración ${ }^{47}$. De varios perfluorocarbonos que se han usado en clínica, el más estudiado es el Perflubron del cual existen dos formas; una radioopaca, que se usa como contraste en estudios de imágenes y una radiolúcida, que sería más útil en clínica ya que se puede evaluar los cambios radiológicos que se producen cuando el producto se utiliza especialmente para ventilación mecánica parcial líquida.

El Perflubron no reacciona con el oxígeno sino que lo transporta disuelto como sucede en el plasma. No es necesaria la combinación con ninguna otra molécula como la hemoglobina lo que resulta en una curva de disociación rectilínea y no sigmoidea por lo que la entrega de $\mathrm{O}_{2}$ es mucho más rápida que la entrega de $\mathrm{O}_{2}$ por la hemoglobina. Su capacidad de transporte de $\mathrm{O}_{2}$ es 3 veces la de la sangre $\left(37-75 \mathrm{ml} / \mathrm{dl} \mathrm{a} 25^{\circ} \mathrm{C}\right)$ y tiene 4 veces más capacidad de transporte de $\mathrm{CO}_{2}$ que de $\mathrm{O}_{2}(122 \text { a } 225 \mathrm{ml} / \mathrm{dl})^{49}$. Dadas las propiedades de estas substancias, se han implementado diversos trabajos en animales de experimentación, induciendo daño pulmonar agudo que lleve a falla respiratoria requiriendo de apoyo ventilatorio mecánico y se les ha ventilado con dos modalidades; 
ventilación líquida total, que requiere de ventiladores especiales y preoxigenación del producto previo al ingreso a la vía aérea y la ventilación líquida parcial, que utiliza ventiladores comunes y asocia la infusión del perfluorocarbono por la conección lateral de la vía inhalatoria y la ventilación con gas. Esta última técnica ha sido la más estudiada y utilizada en clínica humana. La técnica se ha utilizado en recién nacidos con SDRA, SDRN y en adultos con SDRA. En general, se ha obtenido un aumento en la compliance dinámica, aumento en los índices de oxigenación, disminución de los requerimientos de $\mathrm{O}_{2}$, disminución de la fracción de shunt dentro de las primeras horas de instalación de la terapia y disminución significativa de la progresión del SDRA. Los pacientes en varios estudios han requerido de aspiración frecuente por el desplazamiento de los detritus alveolares hacia la vía aérea central en ocasiones con obstrucción del tubo endotraqueal ${ }^{48,49}$. En un estudio controlado en un grupo importante de 90 adultos, se comparó los resultados de días libres de ventilación mecánica y mortalidad entre 65 pacientes que recibieron ventilación parcial líquida y 25 que recibieron ventilación mecánica tradicional. No hubo diferencias significativas entre los grupos, con un número de efectos adversos ligeramente mayor pero no significativo en el grupo de ventilación líquida especialmente neumotórax pero, en el subgrupo de individuos menores de 55 años hubo diferencias significativas en el número de días libres de ventilación mecánica: 8,1 en ventilación líquida contra 3,2 en ventilación tradicional y en mortalidad: $24 \%$ para ventilación líquida contra $42 \%$ en ventilación tradicional ${ }^{50}$. En todo caso, el interés por la ventilación líquida decayó una vez que se publicaron las conclusiones del trabajo de la 'ARDS Net' que demostró una disminución de un $22 \%$ de la mortalidad en el SDRA con el cambio de los parámetros ventilatorios tradicionales hacia los de ventilación protectora, con un costo infinitamente menor que el de utilizar perfluorocarbonos $^{51}$. Siguen teniendo interés, las propiedades antiinflamatorias y físicas de estos productos. Así por ejemplo, Croce y cols en 12 pacientes politraumatizados en ventilación parcial líquida, contra pacientes ventilados en forma tradicional, haciendo lavado broncoalveolar demostró, junto con una mejoría de la relación $\mathrm{PaO}_{2} / \mathrm{FiO}_{2}$ de un $10 \%$ a las $48 \mathrm{~h}$, una disminución de los neutrófilos, una disminución de las citoquinas inflamatorias IL-1 e IL-6 y un aumento en la citoquina antiinflamatoria IL-10 solo en el líquido de lavado de los pacientes con ventilación líqui$\mathrm{da}^{52}$. Estudios más recientes demuestran el valor protector del daño anatómico en el pulmón en un modelo experimental de SDRA con ventilación líquida. El puntaje de daño histopatológico en las zonas dependientes del pulmón fue menor en la ventilación líquida al compararlo con la ventilación tradicional al igual que los niveles de 4-HNE (4-hidroxinonenal) un marcador de peroxidasa lipídica fueron menores en este grupo de animales. Es muy probable que, por sus características físicas, el Perflubron utilizado se dispuso en las zonas dependientes del pulmón, determinando un grado de protección mayor en esas regiones ${ }^{53}$. Otro experimento en daño pulmonar inducido por la instilación de meconio compara los resultados de la ventilación con alta frecuencia oscilatoria contra el uso de surfactante sintético y Perflubron, en la limpieza de las alteraciones observadas demostrando una mejoría significativa en el intercambio gaseoso al lavar el pulmón con surfactante y con Perflubron ${ }^{54}$.

En el año 2000 Woods demostró en un estudio de laboratorio que el Perflubron atenúa la adhesión de los neutrófilos a células endoteliales activadas al observar que células endoteliales dispuestas en monocapa y preexpuestas al producto mostraban más de un $40 \%$ de reducción en los niveles de E-selectina y en la expresión intracelular de moléculas de adhesión-1 posterior a un estímulo proinflamatorio ${ }^{55}$. Se ha demostrado que la exposición in vitro de macrófagos humanos obtenidos por lavado pulmonar a Perflubron disminuye de manera significativa la producción de peróxido de hidrogeno y la liberación de citoquinas logrando una disminución de la quimiotaxis de neutrófilos frente a estímulos externos luego de $2 \mathrm{~h}$ de exposición sugiriendo un efecto antiinflamatorio potente ${ }^{56}$.

Ahora bien, los macrófagos juegan un papel fundamental en la etiopatogenia de la PAP y no solo por su déficit cuantitativo y funcional sino también en la inflamación asociada a la enfermedad. En efecto, Hirano y colaboradores demostraron que en pacientes con PAP se puede evaluar el grado de inflamación macrofágica midiendo la concentración de óxido nítrico alveolar periférico en el líquido de lavado pulmonar total (CANO $\mathrm{ppb}$ ) definiendo un punto de corte de 5,3 ppb, a diferencia de la $\mathrm{F}_{\mathrm{E}} \mathrm{NO}$ que mide la inflamación a nivel de la vía aérea central, con un punto de corte de $50 \mathrm{ppb}$. Existe una muy buena correlación entre los valores de CANO y los índices de gravedad de la PAP como la DLCO, LDH, diferencia alvéolo-arterial de $\mathrm{PO}_{2}$ y los índices de compromiso en la tomografía computada como también con la mejoría significativa de los mismos luego de un lavado pulmonar total exitoso ${ }^{57}$. 
Wan y cols describen el uso de perflubron para lavado pulmonar luego del fracaso del lavado con solución salina en un recién nacido de 6 semanas, con PAP congénita, con hipoxemia severa, en ventilación mecánica y ECMO (oxigenación por membrana extracorpórea) en el cual observaron un aumento del volumen corriente, de la compliance dinámica y de la $\mathrm{PaO}_{2}$, con una disminución de la diferencia alvéolo-arterial de $\mathrm{PO}_{2}$ que permitió el retiro de ECMO. El paciente falleció un mes más tarde probablemente porque la terapia adecuada no era solo el lavado ${ }^{58}$. Lindemann en 2007 reporta el caso de un niño de 2 meses en falla respiratoria severa cuya biopsia pulmonar demostró relleno alveolar con material proteináceo y macrófagos espumosos que falló al tratamiento de lavado pulmonar total con so- lución salina agravándose la hipoxemia. Se lavó con un perfluorocarbono (perfluorodecalina) y durante el procedimiento, el paciente se mantuvo estable con mejoría de la oxigenación sin requerir de ECMO, pero con progresión del compromiso sistémico que le llevó al óbito. La autopsia fue consistente con un Niemann Pick C2 la cual no tiene hasta el momento un tratamiento específico ${ }^{59}$. Por último, existe el reporte en nuestro medio de Rioseco y colaboradores que demuestra la utilidad del uso de un perfluorocarbono para lavado pulmonar lobar en una paciente con una PAP grave que fracasó al LPT habitual ${ }^{5}$.

De acuerdo a lo que hemos descrito, en la Figura 2 se sintetiza el manejo tanto desde el punto de vista diagnóstico como terapéutico de la PAP.

Finalmente, debemos tener en cuenta que si

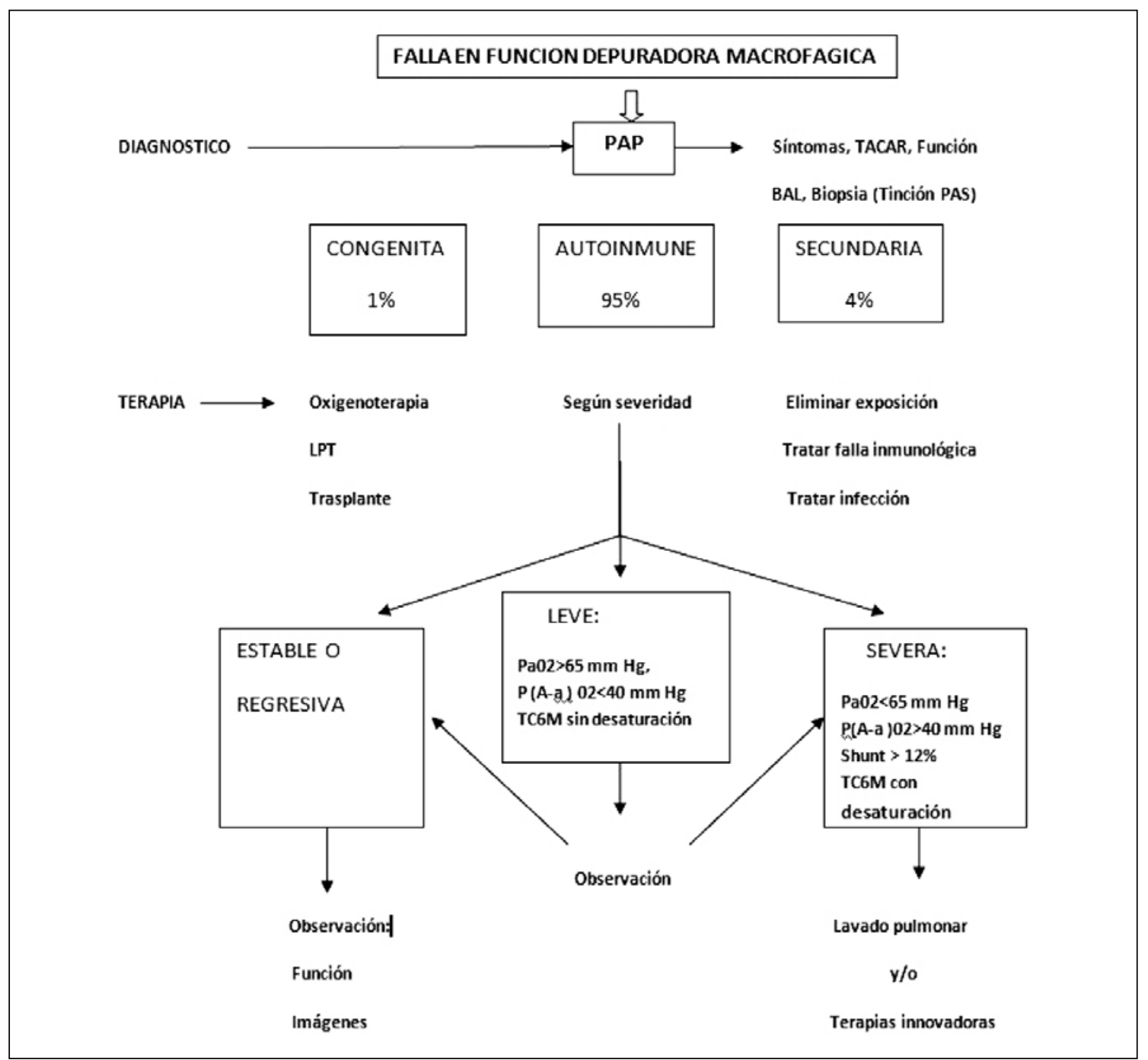

Figura 2. Esquema general del manejo diagnóstico y terapéutico de la Proteinosis Alveolar Pulmonar (PAP); BAL: Lavado broncoalveolar; TACAR: Tomografía axial computarizada de alta resolución; LPT: Lavado pulmonar total; TC6M: Test de caminata de $6 \mathrm{~min}$. 
bien la PAP es un cuadro muy poco probable de encontrar en nuestra práctica clínica habitual, no por ello no debemos tenerlo en cuenta ante un paciente que se presenta con disnea progresiva, insidiosa de un comienzo difícil de establecer, falla respiratoria hipoxémica, infecciones pulmonares no habituales, compromiso funcional y radiológico muy severo y eventualmente fibrosis pulmonar final que lleva a la invalidez. El manejo terapéutico con LPT intenta mitigar la sintomatología y lo logra, pero no es la solución. En la actualidad, existen nuevas formas de lavado pulmonar y terapias que se dirigen más hacia la patogénesis de la enfermedad que a sus consecuencias cuyo blanco podría estar en la modulación de la acción de GM-CSF en la gran mayoría de los enfermos.

\section{Bibliografía}

1.- CARRINGTON JM, HERSHBERGER DM. Pulmonary Alveolar Proteinosis (Updated 2018 March 20) Stat Pearls (Internet) Treasure Island (FL) Stat Pearls publishing. Disponible en: https//www.nlm.bov/books/ NBK482308.

2.- HUARINGA AJ, FRANCIS WH. Pulmonary Alveolar Proteinosis: a case report and world literature review. Respirol Case Rep 2016 13;4(6):e0021. doi:10.1002/ rcr2.201 eCollection 2016 Nov.

3.- PARAM T, FLORES M, BOLBARAN JP, DÍAZ A, ACUÑA JO, OCHOA L. Proteinosis Alveolar Pulmonar, caso clínico. Rev Chil Pedriatr 2007; 78: 176-82. Disponible en: http://dx.doi.org/104067/S037041062007000200009 .

4.- GANDO S, DURÉA R, VILI D, VÁSQUEZ B, LABARCA B, FERNÁNDEZ-BUSSY S. Bilateral lung disease, extensive and diffuse. Diagnosis of PAP by bronchoscopic cryobiopsy. Respir Med Case Rep 2017; 22: $260-2$

5.- RIOSECO P, MUÑOZ S, VIVALDI C, FUICAA, BARI

C. Patrón Crazy Paving en una mujer joven. Hallazgo inesperado y tratamiento no tradicional de un caso de Proteinosis Alveolar Pulmonar. Rev Chil Enferm Respir 2019; 35: 199-206.

6.- ROSEN SH, CASTLEMAN B, LIEBOW AA. Pulmonary Alveolar Proteinosis. N Engl J Med 1958; 258: 1123-42.

7.- DE SANCTIS PM. Pulmonary Alveolar Proteinosis.A review of the findings and theories to date with a disgression on Pneumocistis carinii Pneumonia. BQM 1962; 13: 19-35.

8.- RAMÍREZ J, SCHULTZ RB, DUTTON RE. Pulmonary Alveolar Proteinosis: a new technique and rationale for treatment. Arch Intern Med 1963; 112: 419-31.

9.- RAMÍREZ J, KIEFFER RF, BALL W. Bronchopulmo- nar lavage in man. Ann Intern Med 1965; 63: 819-28.

10.- HAN SH, MALAMPALLI RK. The role of surfactant in Lung Disease and Host Defense against Pulmonary Infections. Ann Amer Thorac Soc 2015; 12: 765-74 doi:10.1513/Annals ATS. 2001411-507FR.

11.- CROUCH E, WRIGHT JR. Surfactants proteins A and D and pulmonary host defense. Annu Rev Physiol 2001; 63: 521-54.

12.- CROUCH E, HARTSHOMK, OFEK J. Collectins and pulmonary innate immunity. Immunol Rev 2000; 173: 52-65.

13.- RUGE CA. Interactions in Peripheral Lungs: Role of Pulmonary Surfactant Components In Alveolar Macrophage Clearance of Nanoparticles, Dissertation Zur Erlangung des Grades des Doktor der Naturwissenchaften der Naturwissenschaftlich-TechnischenFakultat III Chemie,Pharmazie,Bio und Werkstoffwissenschaften der Universitat des Saarlandes 2012.

14.- PÉREZ-GIL J. Structure of pulmonary surfactant membranes and films: The role of proteins and lipid-protein interactions. Biochimica et Biophysica Acta 2008; 1778 : 1676-1695.

15.- WRIGHT JR, CLEMENTS JA. Metabolism and turnover of lung surfactant. Am Rev Respir Dis 1987; 136: 426-44.

16.- ANDREEVA AV, KUTUZOV M, VOYNO-YASENETSKAYA T. Regulation of Surfactant Secretion in Alveolar Type II Cells. Am J Physiol Lung Cell Mol Physiol 2007; 293: L259-71.

17.- WHITSETT JA, WERT S, WEAVER T. Diseases of Pulmonary Surfactant Homeostasis. Annu Rev Pathol 2015; 10: 371-93. Doi:10.1146/annurevpathol-012513-104644.

18.- SUZUKI T, SAKAGAMI T, YOUNG LR, CAREY BC, WOOD RE, LUISETTI M, et al. Hereditary Pulmonary Alveolar Proteinosis: pathogenesis, presentation, diagnosis and therapy. Am J Respir Crit Care Med 2010; 182: 1292-304.

19.- ISHII H, TAZAWA R, KANEKO C, SARAYA T, INOUE $\mathrm{Y}$, HAMANO E, et al. Clinical features of secondary Pulmonary Alveolar Proteinosis: Pre mortem cases in Japan. Eur Respir J 2011; 37: 465-8 doi: 10.1183/09031936.00092910.

20.- BORIE R, DANEL MP, DEBRAY C. TAILLE C, DOMBRET MC, AUBIER M, et al. Pulmonary Alveolar Proteinosis. Eur Respir J 2011; 120: 98-107.

21.- LISON D, LALOY J, CORAZZARI I, MULLER J, RABOLLI B, PANIN N, et al. Sintered Indium-tin-oxide (ITO) particles: A new pneumotoxic entity. Toxicol Sci 2009; 108: 472-81.

22.- INOUE Y, TRAPNELL BC, TAZAWA R, ARAL T, TAKADA T, HIZAWA N, et al. Characteristics of a large cohort of autoimmune Pulmonary Alveolar Proteinosis patients. Am J Respir Crit Care Med 2008; 177 : 752-62.

23.- PUNATAR AD, KUSNE S, BLAIR JE,SEVILLE 
MT,VIKRAM HR. Oportunistic infections in patients with Pulmonary Alveolar Proteinosis. J Infect 2012; 65: 173-9.

24.- SEYMUR JF, PRESNEILL JJ. PAP: progress in the first 44 years. Am J Respir Crit Care Med 2002; 116: 215-35.

25.- JOHKOH T, ITOH H, MULLER NL. Ishikado K, Nakamura H, Ikezoe J, et al. Crazy-paving appearance at thin-section CT: spectrum of disease and pathologic findings. Radiology 1999; 211: 155-60.

26.- HOLBERT JM, COSTELLO P, LI WEI, HOFFMAN RM, ROGERS RM. Features of Pulmonary Alveolar Proteinosis. Am J Roentgenol 2001; 176: 1287-94

27.- KEDIA RK, SULLIVAN A, STEPHENS M, ALLEN MB. A breathless female: case for diagnosis. Eur Resp J 1999; 13: 207-9.

28.- DELANUR C, ZAHARIE AM, SERBESCU A, MIMINITU F, DUMITRU F, ARGHIR OC. Analysis of bronchoalveolar lavage in a first romanian Pulmonary Alveolar Proteinosis cohort. Rom J Morphol Embryol 2016; 57 (2S): 737-43.

29.- SALAÜN M, ROUSSEL F, HAUSS PA, LASHKAR $\mathrm{S}$, THIBERVILLE L. In Vivo imaging of PAP using confocal endomicroscopy. Eur Respir J 2010; 36: 451-3. doi: 10.1183/09031936.00194509.

30.- CAMPO I, LUISETTI M, GRIESE M, TRAPNELL BC; BONELLA F, GRUTTERS J, et al. Whole lung lavage for pulmonary alveolar proteinosis: A global survey for current practices and procedures. Orphanet J Rare Dis 2016, 11: 115. doi 101186/s13023-016-0497-9.

31.- BONELLA F, BAUER PC, GRIESE M. Pulmonary Alveolar Proteinosis: new insights form a single center cohort of 70 patients. Respir Med 2011; 105: 1908-16.

32.- SHAH PL, HANSELL D, LAWSON PR, REID KB, MORGAN C. Pulmonary Alveolar Proteinosis: clinical aspects and current concepts on pathogenesis. Thorax 2000; 55: 67-77.

33.- KAVURU MS, SULLIVAN EJ, PICCIN R, THOMASSEN MJ, STOLLER JK. Exogenous granulocytemacrophage colony stimulating factor administration for Pulmonary Alveolar Proteinosis. Am J Respir Crit Care Med 2000; 161: 1143-8

34.- WYLAM ME, TEN R, PRAKASH UB, NADROUS HF, CLAWSON ML, ANDERSON PM. Aerosol granulocyte macrophage-colony stimulating factor for Pulmonary Alveolar Proteinosis. Eur Respir J 2006; 27 : 585-93.

35.- TAZAWA R, TRAPNELL BC, INOUE Y, ARAI T, TAKADA T, NASUHARA Y, et al. Inhaled granulocytemacrophage colony stimulating factor as therapy for Pulmonary Alveolar Proteinosis. Am J Respir Crit Care Med 2010; 181: 1345-54.

36.- RODRÍGUEZ-PORTAL JL. Tratamiento de la proteinosis alveolar primaria del adulto. Arch Bronconeumol 2015; 51: 311-68. doi:10.1016/j.arbres.2015.02.003.

37.- BORIE R, DEBRAY MP, LAINE C, AUBIER M, CRESTANI B. Rituximab therapy in autoimmune
Pulmonary Alveolar Proteinosis. Eur Respir J 2009; 33:1503-6.

38.- AMITAKL A, DUX S, SHRITIT D. Therapeutic effectiveness of rituximab in a patient with unresponsive autoimmune PAP. Thorax 2010; 65: 1025-6.

39.- KAVURU MS, MALU A, MARSHALL I, BAMA BP, MEZIANE M, HUIZAR I, et al. An open label trial of rituximab therapy in Pulmonary Alveolar Proteinosis. Eur Respir J 2011; 38: 1361-7.

40.- KAVURU MS, BONFIELD TJ,THOMASSEN MJ. Plasmapheresis, GM-CSF and alveolar proteinosis. Am J Respir Crit Care Med 2003; 167: 1036.

41.- LUISETTI M, PEROTTI C, CAMPO I, MARIANI F, POZZI E, TRAPNELL BC. Plasmapheresis for treatment of Pulmonary Alveolar Proteinosis. Eur Respir J 2009; 33: 1220-2.

42.- GARBER B, ALBORES J, WANG T, NEVILLE TH. A plasmapheresis protocol for refractory Pulmonary Alveolar Proteinosis. Lung 2015; 193: 209-11. doi: 10.1007/s00408-014-9678.

43.- DOERSCHUK CM. Pulmonary alveolar proteinosis and macrophage transplantation. N Engl J Med 2015; 372 1762-4. doi:10.1056/NEJMcibr1413035.

44.- SUZUKI T, ARUMUGAM P, SAKAGAMI T, LACHMANN N, CHALK C, SALLESE A, et al. Pulmonary macrophage transplantation therapy Nature 2014; 514 (7523): 450-4.

45.- HAPPLE C, LACHMANN N, ACKERMANN N, MIRENSKA A, GÖHRING G, THOMAY K, et al. Pulmonary transplantation of human induced pluripotent stem-cell-derived macrophages ameliorates Pulmonary Alveolar Proteinosis. Am J Respir Crit Care Med 2018; 198: 350-60. doi:10.1164/rccm,201708-1562OC.

46.- HUDDLESTON CB, BLOCH JB, SWEET SC, DE LA MORENA M, PATTERSON GA, MENDELOFF EM. Lung transplantation in children. Ann Surg 2002; 236: 270-6.

47.- BONELLA F, BAUER PC, GRIESE M. Wash-out kinetics and efficacy of a modified lavage technique for alveolar proteinosis. Eur Respir J 2004; 23: 503-5.

48.- REICKERT C, PRANIKO T, OVERBECK M, KAZEROONI E, MASSEY K, BARTLETT R, et al. The pulmonary and systemic distribution and elimination of perflubron from adult patients treated with partial liquid ventilation. Chest 2001; 119: 515-22.

49.- SPAHN DR, KOCIAN R. Artificial oxygen carriers; Status in 2005. Current Pharmaceutical Design 2005; 31: 4099-114

50.- HIRSCHIL RB. Liquid ventilation for neonatal respiratory failure. In: Manual of neonate respiratory care. Ed SM Donn \& SK Sinha. Fourth edition. Springer 2017. Págs 551-4. Doi:10.1007/978-3-319-39839-6.

51.- PATANKA, NIKHIL AL-IBRAHIM O. Use of perflubron for pulmonary lavage in severe pediatrics ARDS. Crit Care Med 2015; 43: 180. doi: 10.1097/01. ccm.0000474544. 
52.- KACMARECK R,WIEDEMANN HP, LAVIN PT. Partial liquid ventilation in adults with acute respiratory distress syndrome. Am J Crit Care Med 2006; 173: 882-9.

53.- ARDS NETWORK, BROWER RG, MATTHAY MA, MORRIS A, SCHOENFELD D, THOMPSON BT, WHEELER A. Ventilation with lower tidal volumes compared with traditional volumes for acute lung injury and the Acute Respiratory Distress Syndrome. N Engl J Med 2000; 342: 1301-8.

54.- CROCE MA, FABIAN TC, PATTON JH Jr, MELTON SM, MOORE M, TREMTHEM LL. Partial liquid ventilation decreases the inflammatory response in the alveolar environment of trauma patients. J Trauma 1998; 45: 273-80.

55.- VIANA M, SARGENTELLI G, ARRUDA A, WIRYAWAN B, ROTTA AT. The impact of mechanical ventilation strategies that minimize atelectrauma in an experimental model of acute lung injury. J Pediatr 2004; 80: $186-96$.

56.- SCHÖSSLER DL, VELDMAN A, FISCHER D. Comparison of effects of Perflubron and Surfactant lavage on pulmonary gas exchange in a piglet model of meconium aspiration. Biol Neonate 2002; 81: 126-31.

57.- WOODS C, NESLUND G, KORNBRUST E, FLAIM SF. Perflubron attenuates neutrophil adhesion to activated endothelial cells in vitro. Am J Physiol Lung Cell Mol Physiol 2000; 278: L1008-17.

58.- ANZUETO A, MELO J, CAVIEDES I. Ventilación líquida. Med Intensiva 2001; 25: 200-7.

59.- HIRANO T, OHKOUCHI S, TODE N, KOBAYASHI M, ONO M, SATOH T, et al. Peripheral alveolar nitric oxide concentration reflects alveolar inflammation in autoimmune pulmonary alveolar proteinosis. Eur Respir J Open Res 2018; 4: 00071-2017 [doi:. org/10.1183/23120541.00071-2017] disponible en: https://openres.ersjournals.com/content/4/1/00071-2017

60.- WAN CHT, LEWIS D, HISCHL RB. Liquid ventilation in an infant with pulmonary alveolar proteinosis. Pediatric pulmonology 1998; 26: 283-6.

61.- LINDEMANN R, RAJKA T, HENRICHSEN T, VINORUM OG, DE LANGE C, ERICHSEN A, et al. Bronchioalveolar lavage with perfluorochemical liquid during conventional ventilation. Ped Crit Care Med 2007; 8: 486-8. doi:10.1097/01.PCC0000282757.25347.6C.

Correspondencia a:

Dr. Patricio Rioseco S.

Alto Horno 777 Talcahuano.Chile.

Email: parioseco@gmail.com 